\title{
Supplementary Protocol
}

\section{Cryo-OrbiSIMS for 3D molecular imaging of a bacterial biofilm in its native state}

Junting Zhang ${ }^{1}$, James Brown², David J. Scurr ${ }^{3}$, Anwen Bullen ${ }^{4,5}$, Kirsty MacLellanGibson 4 , Paul Williams², Morgan R. Alexander ${ }^{3}$, Kim R. Hardie², lan S. Gilmore*1,3 and Paulina D. Rakowska ${ }^{1}$

${ }^{1}$ National Physical Laboratory, NiCE-MSI, Hampton Rd, Teddington, Middlesex, UK.

2 Biodiscovery Institute and School of Life Sciences, The University of Nottingham, University Park, Nottingham, UK.

${ }^{3}$ School of Pharmacy, The University of Nottingham, University Park, Nottingham, UK.

${ }^{4}$ National Institute for Biological Standards and Controls, Biological Imaging Group, Blanche Ln, South Mimms, Potters Bar, UK.

${ }^{5}$ UCL Ear Institute, 332 Grays Inn Rd, London, UK.

${ }^{*}$ Corresponding author: ian.gilmore@npl.co.uk

\section{Table of Contents}

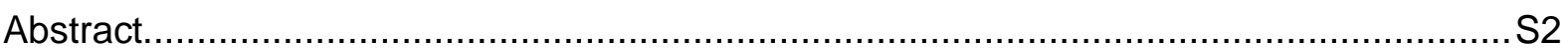

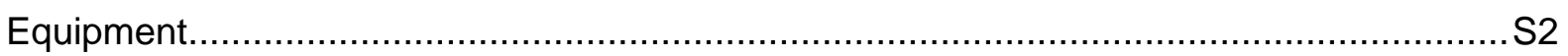

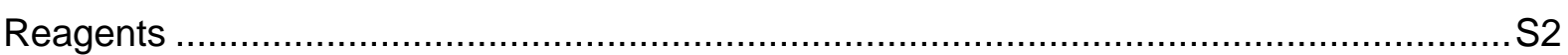

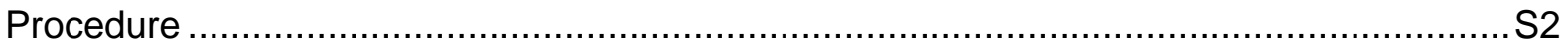

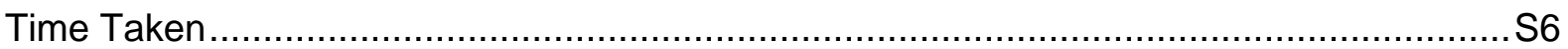

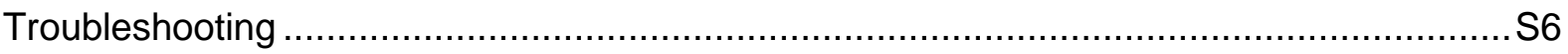

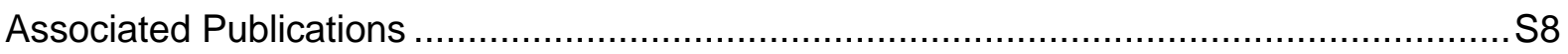

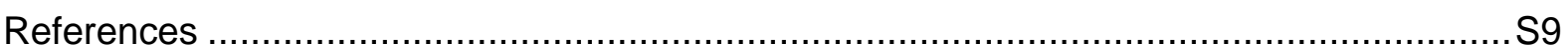




\begin{abstract}
A step-by-step protocol for imaging of frozen-hydrated bacterial biofilm or bacterial pellet using Cryo-OrbiSIMS is provided. The protocol describes requirements for growing bacterial and biofilm, high pressure freezing of samples, transferring samples by cryo-transfer system and set-up of Cryo-OrbiSIMS.
\end{abstract}

\title{
Equipment
}

- High pressure freezer (Leica EM ICE, Germany)

- Cryo-transfer system (Leica EM VCM and Leica EM VCT500, Germany)

- 3D OrbiSIMS (Hybrid SIMS, IONTOF GmbH, Germany)

\section{Reagents}

- Lysogeny Broth (LB) agar (Sigma-Aldrich)

- LB media (Sigma-Aldrich)

- PBS (Sigma-Aldrich)

- FAB medium (Lab M)

- Ethanol (Fisher Scientific)

- Ammonium Formate (Sigma-Aldrich)

- Water (Fisher Scientific)

- Silver (Ag) foil (the exact type is not critical)

\section{Procedure}

1. Bacteria and biofilm growth

a. Streak bacteria on solid media (LB agar plates) to obtain single, well-defined colonies

b. Incubate plate overnight at $37^{\circ} \mathrm{C}$ 

c. In the morning, remove plate from incubator
d. Use a single colony to inoculate $5 \mathrm{ml}$ fresh LB medium
e. Incubate overnight with shaking at $37^{\circ} \mathrm{C}$
f. Take $1 \mathrm{ml}$ culture, pellet in centrifuge (6000rpm, $2 \mathrm{~min}$ )
g. Resuspend pellet in $1 \mathrm{ml}$ PBS
h. Measure $\mathrm{OD}_{600}$ of culture

i. Dilute culture to $\mathrm{OD}_{600}=0.05$ in $\mathrm{FAB}$ medium and $30 \mathrm{mM}$ glucose.

j. Biofilms were directly grown on $3 \mathrm{~mm}$ aluminium specimen carrier (planchette) flat face over 48 hs using a rotary flow system. Media was replaced after $24 \mathrm{~h}$.

k. After $48+h$ carefully wash the biofilms with $150 \mathrm{mM}$ ammonium formate to remove non-adherent planktonic bacteria

\section{High Pressure Freezing of samples}

a. After sample washing, gently remove the Al specimen carrier (planchette) Type B for assembly into a sandwich for high pressure freezing;

b. Place a cleaned Al specimen carrier (planchette) type A $0.1 \mathrm{~mm}$ depth-Indentation side up and fill it with $150 \mathrm{mM}$ ammonium formate solution, place the Al specimen carrier (planchette) type B flat side down onto the ammonium formate solution (Fig. 1);

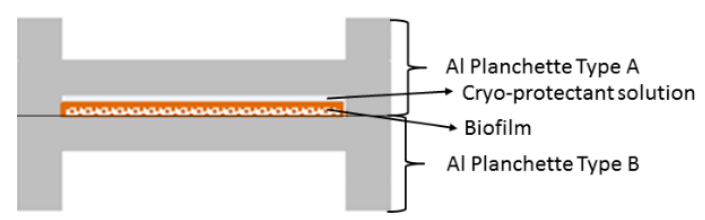

Fig. 1 Schematic to show planchette sandwiches of biofilm for HPF

c. Freeze according to high pressure freezer protocols for your high-pressure freezer.

d. Transfer and store samples in liquid nitrogen until transfer to Cryo-OrbiSIMS. 
a. Mount samples on specimen holders (Fig. 2) in the preparation bath of Leica EM VCM.

b. Transfer samples from preparation bath to manual transfer shuttle (Leica EM VCT500) and detach from VCM. Samples will stay in the cooled and evacuated transfer shuttle before transferring to Cryo-OrbiSIMS (Fig. 3).

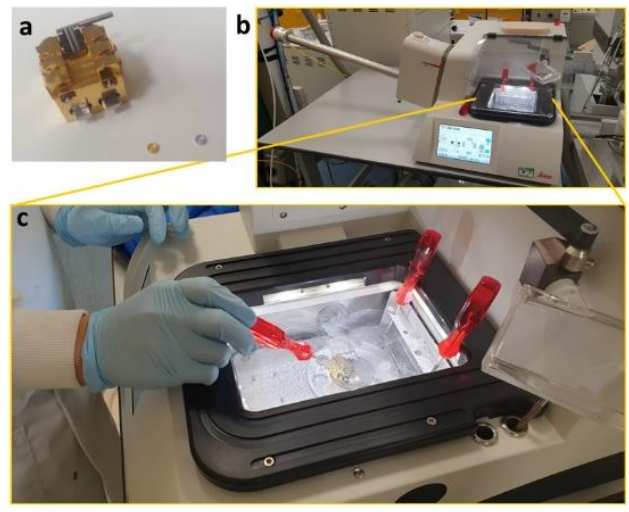

Fig. 2. Picture of step 3a. (a) Picture of one kind of specimen holders; (b) Picture of Leica EM VCT500 shuttle connected to Leica EM VCM; (c) Zoom-in picture of sample preparation bath of Leica EM VCM

a
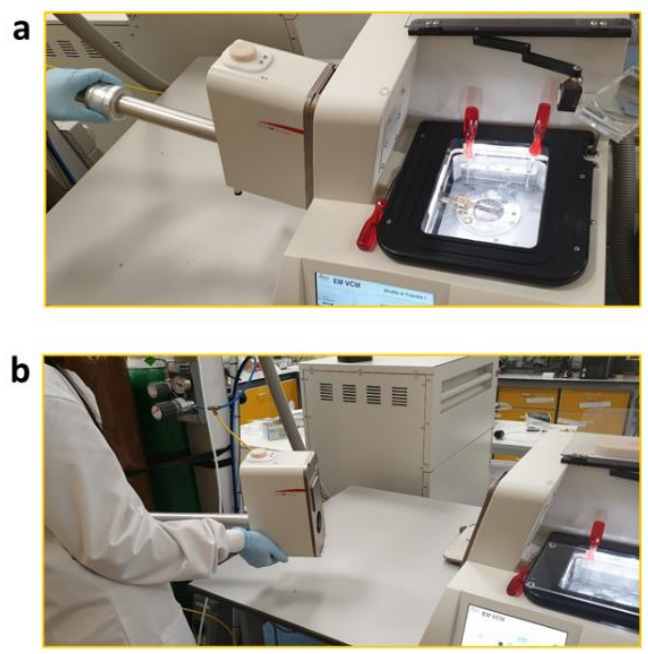

Fig. 3. Picture of step 3b. (a) Picture of transferring samples from preparation bath to manual transfer shuttle; (b) Picture of Leica EM VCT500 shuttle disconnected to Leica EM VCM; 
4. Cryo-OrbiSIMS set up (The Cryo-OrbiSIMS installed in University of Nottingham (Uni Nott) is a little bit different from the one installed in NPL. Here covers two systems)

a. Set up all the required settings in 3D OrbiSIMS and calibrate Orbitrap MS

b. Move heat-cool sample holder (Fig. 4) to the cooling point of load lock (LL) and check the temperature

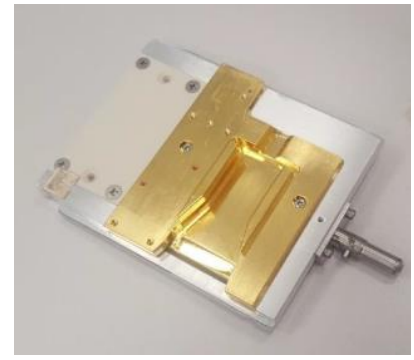

Fig. 4. Picture of heat-cool sample holder (NPL)

C. Insert LL cold finger and tighten (not needed in Uni Nott one)

d. Turn on pumps to the LN2 -LL dewar and main dewar. Wait until temperature cooling down to $-145^{\circ} \mathrm{C}$

e. Remove cold finger and tighten (not needed in Uni Nott one)

f. Move sample to sample holder in LL by using transfer shuttle (Leica EM VCT500) and attaching it to the dock of 3D OrbiSIMS. Transfer the specimen holder to the sample holder in LL (Fig. 5).

a

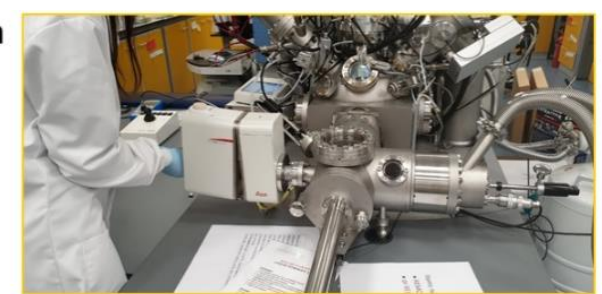

b

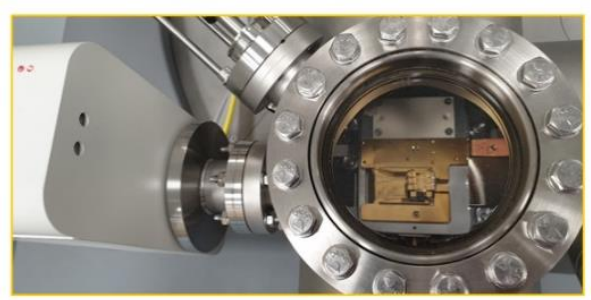

Fig. 5. Picture of step 4f. (a) Picture of attaching transfer shuttle to the dock of 3D OrbiSIMS;

(b) Picture of specimen holder with samples inserted into a pre-cooled SIMS sample holder. 
g. Move the sample holder from LL to pre-cooled main chamber. Load the heatcool.shi file in the navigator and Specify the cooling status: Navigator --> cooling menu --> specific status. In the navigator's positions list: go to "work with cooling". The sample holder will rotate 90 degree, move to the cold finger, move up, pick up the cold finger, then move back to the sample position. (not needed in Uni Nott one) h. Analyze sample. Acquire mass spectrum, depth profile and image following the protocol in ref. 1.

\section{Time Taken}

Biofilm sample preparation takes 3-5 days which depends on which stage of biofilm be studied. High pressure freezing takes 1-2 h. Setting up EM VCM system and loading samples in EM VCM take 30-60 mins. Cryo 3D OrbiSIMS takes 1-2 $\mathrm{h}$ to cool down.

\section{Troubleshooting}

Step 1j. The Al specimen carriers need to be cleaned in ethanol and re-sterilised under the cabinets UV light for a minimum of $1 \mathrm{~h}$, preferably overnight. Al specimen carriers were selected here because biofilm could grow well on this substrate (Supplementary Figure 2) and its conductivity helps to reduce the charging issue in SIMS.

Step 1k. The two different ways to wash biofilms are:

a. Cut the tip off a pipette and use to wash the biofilm carefully with $150 \mathrm{mM}$ ammonium formate solution (pipette up and down slowly once, taking care not to disturb the biofilm); Repeat twice (three washes in total).

b. Using sterilised tweezers carefully dip the coverslip or disk in $150 \mathrm{mM}$ ammonium formate solution to remove non-adherent cells; Repeat up to three times to wash the biofilm. 
Step $2 b$. Assemble specimen carriers as fast as possible to avoid air-drying of samples.

Make sure that there are no air-bubble when filling sample carriers with $150 \mathrm{mM}$ ammonium formate solution. Here, ammonium formate solution was used as cryo-protectant because it's more compatible with SIMS. Other cryo-protectants, which are often used for Cryo-EM sample preparation, were tried such as dextran, 1-hexadecene, BSA, methanol. However, all of them were shown either suppressing or interfering with the signals from samples.

Step 2b. Al specimen carriers have machine marks (Fig. 6a) on the surface and lead to surface roughness around 1-2 $\mu$ m (Fig. 6b). When doing 3D image reconstruction, we just assume this substrate is flat as comparing with 30-50 $\mu$ m-thickness-biofilm, this roughness would not make big effect.

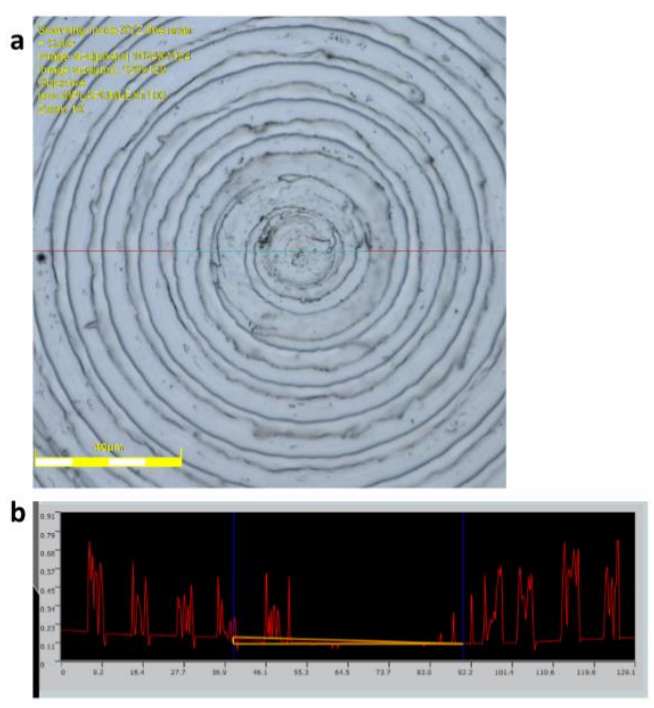

Fig. 6. Laser microscopy of a 3mm (I.D.) Al specimen carrier. (a) Image of centre in $129 \mu \mathrm{m}$ $\times 129 \mu \mathrm{m}$ FoV. (b) Roughness profile of the center line.

Step $2 b$. Ammonium formate was selected as a cryo-protectant as it's much more compatible to SIMS than other four cryo-protectants (dextran, 1-hexadecane, bovine serum albumin (BSA), and methanol). In Supplementary Fig. 3e in which ammonium formate was used, peaks at $\mathrm{m} / \mathrm{z} 523.4720,551.5031,563.5034,591.5348,704.5222,742.4779,770.5092$, 780.4336 which related to DG and PE lipids are shown very clearly. However, the intensity of these peaks at Supplementary Fig. $\mathbf{3 c}$ and $\mathbf{d}$ were quite lower and they're interfered with by 
signals from cryo-protectants at Supplementary Fig. 3a (hexadecane related signals such as $\mathrm{m} / \mathrm{z}$ 253.2161, $\mathrm{m} / \mathrm{z} 477.4665, \mathrm{~m} / \mathrm{z}$ 701.7156, $\mathrm{m} / \mathrm{z}$ 925.9630) and $\mathbf{b}$ (dextran related signals such as $\mathrm{m} / \mathrm{z} 325.1122, \mathrm{~m} / \mathrm{z}$ 487.1648, $\mathrm{m} / \mathrm{z}$ 649.2173).

Step 3. Follow the operating manual of EM VCM and EM VCT 500 for further details. Keep the temperature lower than $-137^{\circ} \mathrm{C}$ and avoid exposing the samples to the air during all the transfer procedures.

Step 4. All the other settings of 3D OrbiSIMS are described in the reference paper ${ }^{1}$.

Step 4e. Make sure temperature is below $-120^{\circ} \mathrm{C}$ to avoid migration of ions. Ions migration were observed when temperature slightly increased from $-120^{\circ} \mathrm{C}$ to $-100^{\circ} \mathrm{C}$ (Figure 7)
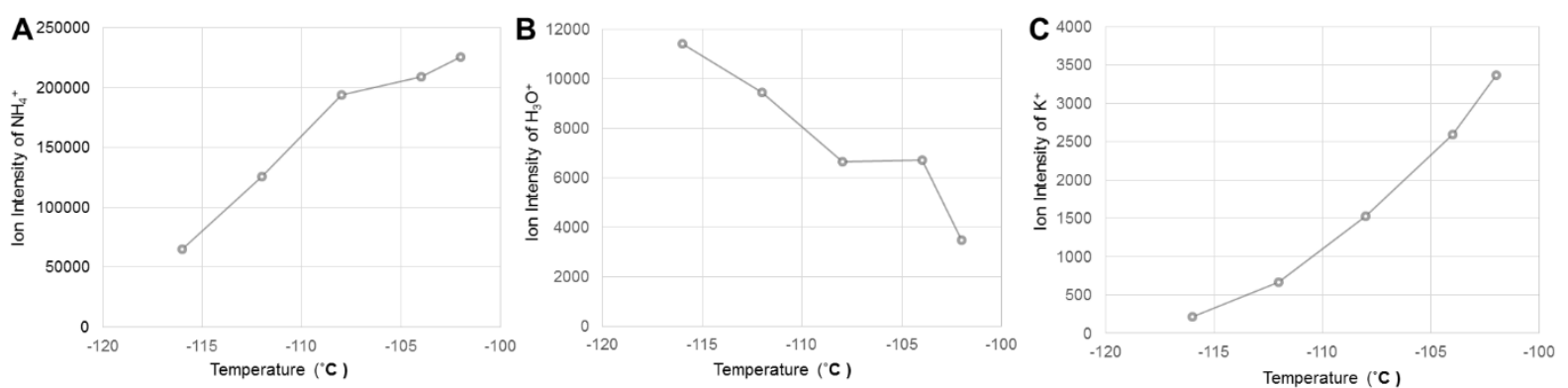

Figure $730 \mathrm{keV} \mathrm{Bi}_{3}{ }^{+}$ToF Signal intensity of different ions $\left(\mathrm{A} . \mathrm{NH}_{4}^{+} ; \mathrm{B} . \mathrm{H}_{3} \mathrm{O}^{+} ; \mathrm{C} . \mathrm{K}^{+}\right)$on the surface versus temperature of sample holder. The migration of salts to the surface (such as $\mathrm{K}^{+}$and $\mathrm{NH}_{4}{ }^{+}$) were observed when temperature increasing from $-120^{\circ} \mathrm{C}$ to $-100^{\circ} \mathrm{C}$ which might be due to water subliming.

\section{Associated Publications}

This protocol was developed as described in the publication "Cryo-OrbiSIMS for 3D molecular imaging of a bacterial biofilm in its native state". The protocol was used to generate Figures 1-5 and supplementary figures of that publication. See the publication and supporting information for expected results. 


\section{References}

1. Passarelli, M. K. et al. The 3D OrbiSIMS-label-free metabolic imaging with subcellular lateral resolution and high mass-resolving power. Nat. Methods 14, 1175$1183(2017)$. 